\title{
Guidelines for return of research results from pediatric genomic studies: deliberations of the Boston Children's Hospital Gene Partnership Informed Cohort Oversight Board
}

\author{
Ingrid A. Holm, MD, MPH ${ }^{1-3}$, Sarah K. Savage, MS1, Robert C. Green, MD, MPH ${ }^{4,5}$, Eric Juengst, $\mathrm{PhD}^{6}$, \\ Amy McGuire, PhD, JD${ }^{7}$, Susan Kornetsky, $\mathrm{MPH}^{8}$, Stephanie J. Brewster, MS', Steven Joffe, MD, MPH ${ }^{9}$ \\ and Patrick Taylor, JD 3,10
}

Purpose: Approaches to return individual results to participants in genomic research variably focus on actionability, duty to share, or participants' preferences. Our group at Boston Children's Hospital has prioritized participants' preferences by implementing the Gene Partnership, a genomic research repository, based on the "Informed Cohort" model that offers return of results in accordance with participant preferences. Recognizing that ethical oversight is essential, the Gene Partnership Informed Cohort Oversight Board was convened in 2009.

Methods: Over 3 years, the Informed Cohort Oversight Board developed guidelines for the return of individual genomic research results.

Results: The Informed Cohort Oversight Board defined its guiding principles as follows: to respect the developing autonomy of pediatric participants and parental decision-making authority by returning results consistent with participants' preferences and to protect participants from harm. Potential harms and strategies to eliminate harm were identified. Guidelines were developed for participant preferences that consider the child's development and family dynamics. The Informed Cohort Oversight Board agreed that to prevent harm, including harms related to interfering with a child's future autonomy, there will be results that should not be returned regardless of participant preferences.

Conclusion: The Informed Cohort Oversight Board developed guidelines for the return of results that respect the preferences of parents, children, and adult participants while seeking to protect against harm.

Genet Med advance online publication 9 January 2014

Key Words: ethics; Informed Cohort Oversight Board; participant preferences; pediatrics; return of individual genomic research results

\section{INTRODUCTION}

The disclosure to participants of individual results that arise in the course of genomic biobank research and other large genomics studies has long been a matter of ethical controversy. ${ }^{1,2}$ Reaching consensus on standards for disclosure of research results has become both more compelling and more complex due to the feasibility of whole-exome/whole-genome sequencing, the vast amounts of data available for interpretation, and the correspondingly greater potential for incidental findings (i.e., genetic findings unrelated to the indication for the sequencing). ${ }^{3}$ Approaches to the return of individual research results vary in the relative weights given, on the one hand, to respect for participant preferences and the potential benefits from the knowledge, and, on the other hand, to concerns about medical and psychosocial harm to participants, their ability to understand the information, and burdens on the research enterprise. Our group has taken an approach to the return of genomic research results that seeks to respect participant preferences, while protecting them from harm, through ethical review and oversight by the Informed Cohort Oversight Board (ICOB). The ICOB is a body separate from, but endorsed by, the Institutional Review Board (IRB), which focuses on providing accurate and comprehensible results in accordance with participant preferences in a manner that minimizes harm. The context for the ICOB is the Gene Partnership (GP), a longitudinal pediatric repository at Boston Children's Hospital (BCH) for the study of the genetic and environmental contributions to childhood health and disease through linkage of biospecimens and genetic data to phenotypic data abstracted from the electronic medical record. GP participants will be allowed to exercise their autonomy by designating their preferences for which research results to receive about themselves and their children. The purpose of this article is to report on the ICOB's guidelines for return of genomic research results in the GP.

General guidelines for return of genomic research results have traditionally focused on the nature of the result itself: analytic validity, clinical validity, actionability, and severity of

'Division of Genetics and Genomics, Boston Children's Hospital, Boston, Massachusetts, USA; ${ }^{2}$ The Manton Center for Orphan Disease Research, Boston Children's Hospital, Boston, Massachusetts, USA; ${ }^{3}$ Department of Pediatrics, Harvard Medical School, Boston, Massachusetts, USA; ${ }^{4}$ Partners Center for Personalized Genetic Medicine, Boston, Massachusetts, USA; ${ }^{5}$ Division of Genetics, Department of Medicine, Brigham and Women's Hospital and Harvard Medical School, Boston, Massachusetts, USA; ${ }^{6}$ NC Center for Bioethics, University of North Carolina at Chapel Hill, Chapel Hill, North Carolina, USA; ${ }^{7}$ Center for Medical Ethics and Health Policy, Baylor College of Medicine, Houston, Texas, USA; ${ }^{8}$ Department of Clinical Investigation, Boston Children's Hospital, Boston, Massachusetts, USA; ${ }^{9}$ Department of Medical Ethics and Health Policy, University of Pennsylvania Perelman School of Medicine, Philadelphia, Pennsylvania, USA; ${ }^{10}$ Children's Hospital Informatics Program, Boston Children’s Hospital, Boston, Massachusetts, USA. Correspondence: Ingrid A. Holm (Ingrid.Holm@childrens.harvard.edu) 
the outcome. Generally, disclosure was discouraged unless all of these elements were met. ${ }^{4}$ Guidelines from several National Institutes of Health-sponsored groups have taken this approach, including the National Heart, Lung, and Blood Institute working group ${ }^{5}$ and the National Cancer Institute working group. ${ }^{6}$ In the clinical context, the American College of Medical Genetics and Genomics (ACMG) has recently made recommendations for return of incidental findings in clinical settings: laboratories should interrogate a specified minimum list of genes of such high clinical significance that the results should be disclosed on the grounds of medical exigency. ${ }^{7}$

There are others, however, who acknowledge that biobanks have a duty to share results, not based on the nature of the findings per se, but because participants have a right to know information that could be important to their medical care., ${ }^{2,8}$ Indeed, studies show that not only do participants desire to receive individual research results, ${ }^{9,10}$ but parents of pediatric participants in fact often desire to receive all research results, irrespective of "clinical utility" or "actionability." ${ }^{11}$ Finally, some commentators ground investigators' responsibilities in duties of ancillary care, defined as "that which goes beyond the requirements of scientific validity, safety, keeping promises, or rectifying injuries."12

The preference-based approach that we have taken is also grounded on the view that participants have a moral claim for results but goes a step further by allowing them to modulate that claim according to their own assessments of their interests. This approach places a high value on participant autonomy and the right to know, or not to know, information that may affect their health. ${ }^{13}$ This approach acknowledges the personal meaning of genomic information to participants ${ }^{1,13,14}$ and is sometimes designated as "personal utility,"15-18 in contrast to actionability, which, although important to consider, may not completely encompass the range of findings that many participants desire.

In 2007, our group proposed the implementation of a preference-based approach to return of individual results in large genomic studies that maximizes participant autonomy: the "Informed Cohort" model. ${ }^{3}$ A key feature of the Informed Cohort is the ability of participants to set their preferences for return of individual research results and to change them over time. This model evolved into the three-dimensional "Multidimensional Results Reporting" model, ${ }^{1}$ which incorporates participant preferences, communicability of the result (i.e., how likely it is that the information contained in the result will be understood by the participant), and the significance of the result (analytic validity, clinical validity, and possibility for medical intervention) into the decision-making process for return of individual genomic information. The Informed Cohort model hypothesizes that respecting participant autonomy by taking into account personal preferences for results provides the most benefit and least harm to participants. At the same time, oversight by the ICOB is essential to establish a protective framework so that the Informed Cohort can safely return research results to participants while ensuring maximal respect for participant preferences. ${ }^{3}$
The BCH ICOB for the GP was convened in September 2009 (see ref. 19). The GP protocol included the ICOB and was approved by the BCH IRB, which requested that we provide them with ICOB policies and that in the initial phase the deliberations around all results to be returned be brought to the IRB for review.

Results from studies performed using GP samples will be returned by the GP staff. In the initial phase, the IRB stipulated that the results be returned by a genetic counselor, usually over the telephone. In the future, the mode of return of a result will depend on the result and the implication-for some results, telephone contact will be mandatory, whereas for others it would not.

In this article, we report on the deliberations of the ICOB, summarize the process and conclusions of the ICOB over the past 3 years, and report the guidelines for return of genomic research results that stemmed from those deliberations. We anticipate that the guidelines and the underlying principles will serve as a reference point for other institutions facing similar challenges.

\section{The BCH ICOB for the GP \\ MATERIALS AND METHODS}

We convened a multidisciplinary GP ICOB, which included a medical geneticist with expertise in clinical trials studying participant response to return of results, a senior genetic counselor, the BCH IRB director of Clinical Research Compliance, and four ethicists with expertise in genomics, including two trained in law and ethics, a pediatric oncologist, and a former chief of the Ethical, Legal, and Social Implications Branch of the National Center for Human Genome Research at the National Institutes of Health. The ICOB recently added a pediatric geneticist with expertise in genetic diagnostics. The ICOB has been staffed by two genetic counselors, a fellow in informatics and health-care information technology, and administrative personnel.

The ICOB had six face-to-face meetings and numerous conference calls over 3 years. During these meetings and calls, the ICOB performed outreach to understand stakeholder views, studied and contrasted different disclosure paradigms, defined principles to guide disclosure from extensive discussion of test cases, and wrote two policy statements that provide the foundation and context for the culmination of these meetings, calls, and statements: the ICOB Guidelines for Return of Results (Figures 1 and 2).

\section{Guiding principles}

\section{RESULTS}

The charge of the ICOB members was to make decisions based on the Informed Cohort model, in particular creating a framework in which the GP could safely maximize participants' ability to receive results in accord with their preferences. As the first major task, the ICOB formulated its purpose and the principles that should guide its decision making. The ICOB defined as its purpose to ensure (whether in designing infrastructure, suggesting policies, or deciding on appropriate communication in particular cases) that research results are conveyed in 


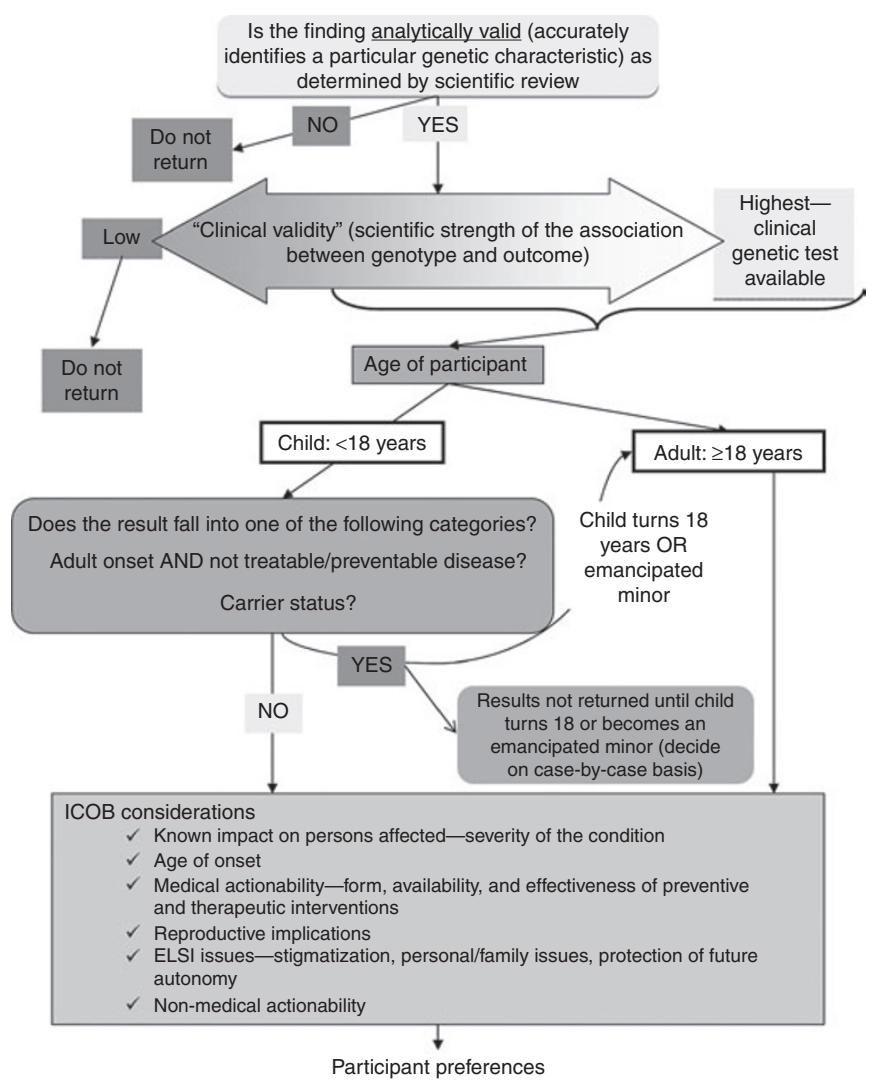

Figure 1 Informed Cohort Oversight Board guidelines flowchart.

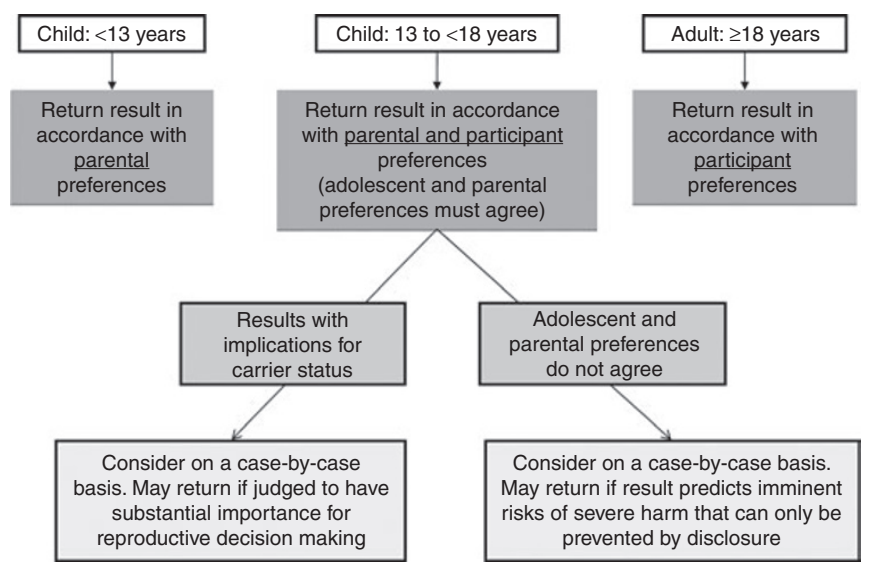

Figure 2 Participant preferences.

a clear, accurate, and understandable way based on educated participant choices and with due regard for minimizing potential harm. To this end, the ICOB defined two fundamental principles that frame the Informed Cohort model and will guide the return of genomic results for the GP:

1. To maximize parent and participant autonomy and choice in deciding about return of research results; and

2. To establish a framework that minimizes the likelihood that disclosure will lead to harm, and to detect and mitigate harm should it occur.

\section{Participant preferences}

The focus on autonomy and choice is in contrast to principles that mandate disclosure of results that are determined to be highly "actionable" regardless of participant choice. Assessing authentic participant preferences may be a challenge; the Informed Cohort model attempts to mitigate this challenge by educating participants on the implications of their preferences and then allowing participants the opportunity to control and change their preferences over time.

Given that the GP is a study of children, an important issue is the tension between respecting a child's future autonomy and the parents' duty to act in ways that are best for, and protect their child. Therefore, the resulting guidelines for participant preferences for return of results consider the child's age and development (Figure 2):

1. GP participants $\geq 18$ years of age who possess decisionmaking capacity: Participants designate their preferences for what type of results they do and do not want to receive.

2. GP participants $<13$ years of age: Parents designate their preferences for what type of results they do and do not want to receive about their child.

3. GP participants 13-17 years of age: Parents and adolescent participants both designate their preferences for what type of results they do and do not want to receive.

\section{Potential conflicts}

Potential conflicts related to the family dynamic inherent in pediatrics may arise within these guidelines for participant preferences.

1. Parents and adolescents disagree regarding their preferences for return of the adolescent's results: Despite the desire to resolve such conflicts, the reality is that the GP is a large study and exploring in-depth the nature of each individual conflict is not scalable. In addition, there is no way to know a priori the nature or frequency of these conflicts. Thus, the ICOB approach to this issue will be prescribed initially and then evolve iteratively with experience. Although each conflict will be considered on a case-bycase basis, given the uncertain ability of the ICOB to resolve the conflict when parents and children disagree, the ICOB believes that the initial appropriate response is to not return a result if the parent and adolescent disagree about disclosure, unless the result predicts imminent risks of severe harm that can be prevented only by disclosure. With experience, as the ICOB starts to understand the nature of these conflicts, it will be better positioned to develop methods to resolve the conflicts, such as family-oriented consultation. Eventually, methods to prevent conflicts from occurring in the first place will be developed, such as asking adolescents and their parents to designate preferences together and discuss the implications of the preferences that they set. 
2. Both parents are involved in the decision about disclosure and express conflicting preferences for results for their child: Although we expect that one parent will usually set the preferences for return of results for their child, both parents may set preferences separately for their child. Many of the same points (above) apply to parental disagreement, and the same approach will be taken, i.e., the initial appropriate response is to not return a result if the parents' preferences regarding the disclosure disagree, unless the result predicts imminent risks of severe harm that can be prevented only by disclosure. Again, each conflict will be considered on a case-by-case basis, and as the ICOB starts to understand the nature of these conflicts, it will be better positioned to develop methods to resolve such conflicts.

\section{Protection from harm}

Harms are detected and mitigated through oversight and ethical review of each case by the ICOB. To guide its review, the ICOB outlined potential harms, which include:

1. Returning results that lack analytic validity;

2. Returning results that lack clinical validity;

3. Returning results to a parent without considering the future autonomy of the child or an incapable adult regarding:

(i) Reproductive choices and

(ii) Adult-onset, nontreatable diseases;

4. Returning results that pose imminent risks of severe harm to families that can be prevented by nondisclosure (e.g., misidentified paternity);

5. Returning results in a manner that is unlikely to be understood by the participant (not easily communicated); and

6. Returning results that are inconsistent with a participant's preferences.

The ICOB then identified strategies for returning results to reduce or eliminate harm:

1. Because meaningful results depend on sound study design, the ability of studies to support individual results should be assessed through independent scientific review to determine whether studies are eligible to use the GP data.

2. Genetic studies to be returned will be performed in a Clinical Laboratory Improvement Amendments-certified laboratory to maximize analytic validity and minimize the possibility of specimen misidentification.

3. The ICOB will consider clinical validity in the return of results. On the one hand, when the result has been incorporated into the evidence-based practice of medicine, or the result has regulatory approval as a diagnostic test, clinical validity may be presumed. However, there will be cases in which the clinical validity is not clear. The ICOB will consider such cases individually, taking a casuistic approach and recognizing that the GP is a research study designed to provide participants the results of new research findings.

4. The result, the proposed modes of communication, and the content of the message will be reviewed to ensure that the results are communicated in a way that is understandable to the participant.

5. Results must be contextualized by what is known and unknown. The uncertain meaning of partial associations should be explained (e.g., providing information on the limitations of the conclusions given lack of knowledge about environmental influences).

6. There must be a process of immediate follow-up and querying of participants, combined with an offer of genetic counselor involvement for further questions or concerns.

7. There should be designated supports for communication of information that may be disappointing or concerning for participants while respecting participant preferences to receive such information.

\section{When the ICOB will override patient preferences}

Although participant autonomy is a fundamental principle, rare cases of clearly avoidable risk of harm related to genetic susceptibility (e.g., juvenile leukemia) may justify overriding a participant's expressed preferences not to receive results. If a parent declines disclosure of discoveries that predict risks of preventable harm to their child, the ICOB will consider disclosing these findings to third parties (treating physicians).

\section{Results that the ICOB will not return}

On the other hand, there may be circumstances under which the ICOB determines that it would not be ethically justified to return results, even if return appeared to be consistent with a participant's preference. For example, the ICOB agreed that there are categories of results that should not be returned to children under 18 years of age, because returning them would interfere with the child's future "sphere of privacy" life planning or implicate the child's future reproductive risks. Such results include adult-onset, nontreatable diseases (e.g., Huntington disease), nonmedical traits (e.g., athletic ability), and carrier status. The ICOB recognized that in rare circumstances, such as when the participant is an emancipated minor or the result is judged to have substantial implications for reproductive decision making for the family, the ICOB may return the result. These will be considered on a case-by-case basis. When the child turns 18 years of age, if he/she consents to continue in the study, he/she will have the option to receive these results if the results are consistent with his/her stated preferences.

In keeping with the ICOB guiding principle to reduce or eliminate harm, the ICOB has identified individual research results NOT to return, regardless of participant preferences. These include results that do not have analytic validity or clinical validity, based on a Scientific Review Group assessment; results that, based on assessment by a genetic counselor, cannot 
be communicated in a clear way; and results that pose imminent risks of severe harm to families if disclosed (e.g., misidentified paternity).

\section{DISCUSSION}

The approach we have taken here is to develop guidelines for the return of genetic research results based on participant autonomy and educated choices, and on minimizing harm. Although our philosophy may not be so different from others, we are operationalizing our philosophy and taking a uniquely casuistic approach by developing guiding principles based on working through case studies. Moving forward, the ICOB will evolve and adapt in response to actual cases, with due regard for precedents set in previously reviewed cases. In this context, and in contrast to other approaches taken, the ICOB infrastructure allows us, for example, to increase participant understanding and to assess for and address participant harm. Our approach is iterative-we will modify standards and definitions based on whether and how they work in practice.

Our approach is also distinctive from that of other investigators because it does not rely on "actionability" as a criterion for return of results. Actionability as a standard for disclosure can be problematic because the definition of "actionability" varies greatly among individuals and for many participants includes "personal utility." Instead, we broaden the spectrum of returnable findings to return to include those with less clear "actionability" and allow participants to decide which results are important for them to receive. This approach is distinct from the approach proposed by Wolf et al., ${ }^{2,8}$ which asks whether there is an information asymmetry regarding information that is critical enough to health to create an ethical or legal duty. We instead incorporate that question within a broader look at participants' best interests as they define them and how we can involve them as knowledgeable partners.

Return of genomic results in the pediatric context has some significant challenges for the ICOB. One of the greatest challenges is the family dynamic, which will potentially lead to conflicts between respect for the child's future autonomy, parental autonomy, potential harms to the child, and potential harms to parents and family members. In particular, results for a child may have implications for other family members, such that protecting a child's future right not to know could lead to harm in family members. The ACMG clinical recommendations ${ }^{7}$ take the view that an incidental finding found in a child in regard to a disease that is not treated in childhood but would be highly treatable in adulthood (e.g., BRCA1 mutations) should be returned due to implications for the parents and other family members. The ICOB does not rule out providing such results to parents. In fact, the ICOB might override parents' preference to receive no results if there was such a result that predicted imminent risk of severe harm in the parent that could be prevented by disclosure in a child. These cases will be considered on a case-by-case basis. In the same manner, a result showing that a female was a carrier for an X-linked disease (e.g., muscular dystrophy) might be considered for return due to the implications for the family. One of the advantages of our approach is that it is dynamic; such cases will be deliberated, and the outcomes of the deliberations will inform future decisions.

One of the ways to balance the child's future autonomy with the family dynamic in the case of adolescents is to allow both the parent and adolescent to set preferences. This model acknowledges the developing child's ability to assert his/her growing autonomy. On the other hand, this may lead to conflicts if a parent and adolescent disagree on the preferences for the child. Because there is a lack of evidence of benefits versus harm, the ICOB is initially taking the conservative approach to not return a result if the parent and adolescent disagree, unless the result predicts imminent risks of severe harm that can be prevented only by disclosure, and to consider each conflict on a case-by-case basis. As the ICOB gains experience and considers these conflicts, it may be able to develop systems and methods to resolve such conflicts.

Recently, the American Academy of Pediatrics and the ACMG issued a joint statement on genetic testing in children. ${ }^{20,21}$ The ICOB policies endorse the American Academy of Pediatrics/ACMG core recommendation not to return information about adult-onset nontreatable diseases but recognize that exceptions to this presumption might need to be made on a case-by-case basis. The ICOB guidelines are also consistent with the American Academy of Pediatrics/ACMG statement, recognizing that there may be tension between the family dynamic and the child's future autonomy. The ICOB approach to adult-onset treatable diseases is not prescriptive. The ICOB will consider the severity of the condition, medical actionability, reproductive implications, personal/family issues, and protection of future autonomy in considering the return of such findings, with due regard for the preferences of the parent and, in the case of adolescents, the child.

Finally, although our model has been developed in a pediatric context, few standards (i.e., adult-onset condition, reproductive implications, and parent-child disagreement) are pediatric specific, so the approach is likely to be useful for studies involving adult participants.

We recognize that there are limitations to the ICOB's guidelines and deliberations. One of the key limitations is that we have not, as of yet, included parents or children in the ICOB deliberations, which will be important to do as the ICOB moves forward. However, we have conducted parent focus groups ${ }^{11}$ and have extensively surveyed parents about return of results in pediatrics and preferences (manuscripts in preparation), and we have taken the responses into account in developing ICOB policies. Another important prerequisite to the return of results will be a method for participants to set their preferences. Our group is working on developing and implementing such a method. Finally, we have not yet returned any results using this model and thus have not fully tested our approach.

\section{Conclusion}

The ICOB for the GP, a genetic repository enabling research on the genetic and environmental factors that influence childhood health and disease, has deliberated for several years and 
developed a carefully crafted approach to maximizing benefit and minimizing harm in the return of individual research results to participants. Recognizing that the participants are children, that the primary receivers of the results are their parents, and that the child is on a developmental trajectory that is evolving toward full autonomy, the ICOB has endeavored to develop guidelines for the return of results that respect the values and desires of parents, children, and adult participants while seeking to protect against harm.

\section{ACKNOWLEDGMENTS}

This work was supported by the National Human Genome Research Institute/National Institutes of Health grants 1RC1HG005491 (I.A.H., principal investigator), 1-R01HG006615 (I.A.H., principal investigator), and HG006500 (R.C.G., principal investigator). The authors thank Isaac S. Kohane and Louis M. Kunkel for their vision, advice, and support in developing the Gene Partnership; Ken Mandl for formulating the concept of the ICOB; and Erin D. Harris for her contribution to this study.

\section{DISCLOSURE}

The authors declare no conflict of interest.

\section{REFERENCES}

1. Kohane IS, Taylor PL. Multidimensional results reporting to participants in genomic studies: getting it right. Sci Trans/ Med. 2010;2:37cm19.

2. Wolf SM, Crock BN, Van Ness B, et al. Managing incidental findings and research results in genomic research involving biobanks and archived data sets. Genet Med 2012;14:361-384.

3. Kohane IS, Mandl KD, Taylor PL, Holm IA, Nigrin DJ, Kunkel LM. Medicine Reestablishing the researcher-patient compact. Science 2007;316: 836-837.

4. Reilly P. When should an investigator share raw data with the subjects? IRB 1980;2:4-5+

5. Fabsitz RR, McGuire A, Sharp RR, et al. Ethical and practical guidelines for reporting genetic research results to study participants: updated guidelines from a National Heart, Lung, and Blood Institute working group. Circ Cardiovasc Genet. 2010;3:574-580.

6. National Cancer Institute. Workshop on Release of Research Results to Participants in Biospecimen Studies. Bethesda, MD, 2010.

7. Green RC, Berg JS, Grody WW, et al. ACMG recommendations for reporting of incidental findings in clinical exome and genome sequencing. Genet Med. 2013;15:565-574.

8. Wolf SM. The past, present, and future of the debate over return of research results and incidental findings. Genet Med 2012;14:355-357.

9. Kaufman D, Murphy J, Scott J, Hudson K. Subjects matter: a survey of public opinions about a large genetic cohort study. Genet Med 2008;10:831-839.

10. Murphy J, Scott J, Kaufman D, Geller G, LeRoy L, Hudson K. Public expectations for return of results from large-cohort genetic research. Am J Bioeth 2008;8: 36-43.

11. Harris ED, Ziniel SI, Amatruda JG, et al. The beliefs, motivations, and expectations of parents who have enrolled their children in a genetic biorepository. Genet Med 2012;14:330-337.

12. Richardson HS, Belsky L. The ancillary-care responsibilities of medical researchers. An ethical framework for thinking about the clinical care that researchers owe their subjects. Hastings Cent Rep 2004;34:2533.

13. Ravitsky $\mathrm{V}$, Wilfond BS. Disclosing individual genetic results to research participants. Am J Bioeth 2006;6:8-17.

14. Rothstein MA. Tiered disclosure options promote the autonomy and well-being of research subjects. Am J Bioeth. 2006;6:20-21; author reply W10-22.

15. Foster MW, Mulvihill JJ, Sharp RR. Evaluating the utility of personal genomic information. Genet Med 2009;11:570-574.

16. Grosse SD, Kalman L, Khoury MJ. Evaluation of the validity and utility of genetic testing for rare diseases. Adv Exp Med Bio/ 2010;686:115-131.

17. Grosse SD, McBride CM, Evans JP, Khoury MJ. Personal utility and genomic information: look before you leap. Genet Med 2009;11:575-576.

18. Khoury MJ, McBride CM, Schully SD, et al.; Centers for Disease Control and Prevention. The Scientific Foundation for personal genomics: recommendations from a National Institutes of Health-Centers for Disease Control and Prevention multidisciplinary workshop. Genet Med 2009;11:559-567.

19. Holm IA, Taylor PL. The Informed Cohort Oversight Board: from values to architecture. Minn. J. L. Sci. \& Tech. 2012;13:669-690.

20. Committee on B. Ethical issues with genetic testing in pediatrics. Pediatrics 2001;107:1451-1455.

21. Ross LF, Ross LF, Saal HM, David KL, Anderson RR; American Academy of Pediatrics; American College of Medical Genetics and Genomics. Technical report: Ethical and policy issues in genetic testing and screening of children. Genet Med 2013;15:234-245. 